\title{
Homogenisation of a chemical degradation mechanism inducing an evolving microstructure
}

\author{
Malte A. Peter ${ }^{a}$, \\ ${ }^{a}$ Centre for Industrial Mathematics, FB 3, University of Bremen, Postfach 330 440, 28334 Bremen, Germany \\ Phone: +4942121863844 , Fax: +494212189406
}

\begin{abstract}
We consider a reaction-diffusion problem in a porous medium, where the reaction causes a local increase or decrease of volume of the solid matrix. For the homogenisation of the resulting system of equations, we employ the method of homogenisation in domains with evolving microstructure. The functions describing the evolution of the microstructure are related to the reaction-diffusion process.
\end{abstract}

\section{Résumé}

Homogénéisation d'un mécanisme du effritement chimique qui cause une évolution de la microstructure. On considère un problème de la réaction et la diffusion dans un milieu poreux, où la réaction cause une augmentation ou une réduction du volume de la matrix solide. Par l'homogénéisation du système d'équations résultants on emploie la méthode d'homogénéisation dans des domaines avec évolution de la micro-structure. Les fonctions que décrivent l'évolution de la micro-structure ont relié au processus de la réaction et la diffusion.

Key words: Homogenization ; multiscale approach ; reaction-diffusion; concrete carbonation ; porous media

Mots-clés : Homogénéisation; approche multiéchelle; reaction et diffusion; carbonatation du beton; milieu poreux

\section{Introduction}

We consider a reaction-diffusion problem involving two species in a porous medium $\Omega$ made up of pore air $\Omega^{\mathrm{a}}$, pore water $\Omega^{\mathrm{w}}$ and solid matrix $\Omega^{\mathrm{s}}$. One species, A, diffuses through the pore air and dissolves in

Email address: mpeter@math.uni-bremen.de (Malte A. Peter). 
the pore water. Another species, B, is part of the solid matrix and dissolves into the pore water. The two species may participate in a reaction of the form

$$
\mathrm{A}+\mathrm{B} \longrightarrow \mathrm{C}+\ldots
$$

where the reaction product $\mathrm{C}$ is of low solubility and (after precipitation) can hence be associated with the solid matrix again. The special feature of the problem under consideration is that owing to different molar densities of $\mathrm{B}$ and $\mathrm{C}$, the reaction causes a local growing or shrinking of the solid matrix. In turn, the pore air and pore water change. Therefore, the total volume occupied by the porous medium $\Omega$ is time-independent while its parts, $\Omega^{\mathrm{a}}, \Omega^{\mathrm{w}}$ and $\Omega^{\mathrm{s}}$, may evolve owing to the conversion of $\mathrm{B}$ to $\mathrm{C}$.

One particular application exhibiting these features is concrete carbonation, where the reactant $\mathrm{A}$ is atmospheric carbon dioxide, which reacts with certain concrete constituents, mainly calcium hydroxide (B). Calcium carbonate (C) is produced in this reaction, which causes a decrease of $\mathrm{pH}$ in the concrete and an increase of volume of solid matrix since the calcium carbonate takes up more volume than the calcium hydroxide. The drop in $\mathrm{pH}$ facilitates the corrosion of reinforcing steel bars and thus decreases the service-life of the structure. More information about concrete carbonation can be found in $[1,2,3]$, e.g., and in $[4,5]$ in the context of multi-scale approaches.

We present a coupled reaction-diffusion system modelling the concentration of $\mathrm{A}$ in the two evolving domains occupied by pore air and pore water. Making use of the method of homogenisation in domains with evolving microstructure presented in [6], we use periodic homogenisation techniques, cf. $[7,8]$ e.g., to find the macroscopic system of equations. In order to focus on the evolution induced by the reaction (1) and to keep the problem similar to the abstract problem presented in [6], we assume the reaction rate to be given and we do not explicitly consider the concentration of $\mathrm{B}$ or $\mathrm{C}$. The problem under consideration can be viewed as a starting point for more complex reaction scenarios including an unknown for the concentration of B as well as non-linear concentration-dependent reaction rates, such as those considered in $[9]$.

\section{The microscopic reaction-diffusion system}

In order to upscale the problem using periodic homogenisation techniques, we employ the ideas of homogenisation in domains with evolving microstructure described in [6], i.e. it is assumed that there exists a reference configuration, which is $\varepsilon$-periodic. By this, we mean that there exists a reference cell $Y=(0,1)^{N}$ with $\bar{Y}=\bar{Z}^{\mathrm{a}} \cup \bar{Z}^{\mathrm{w}} \cup \bar{Z}^{\mathrm{s}}$ and $Z^{\mathrm{a}} \cap Z^{\mathrm{w}} \cap Z^{\mathrm{s}}=\emptyset$, such that $\Omega_{*}^{i}=\bar{\Omega} \cap \operatorname{int} \bigcup_{k \in \mathbb{Z}^{N}} \varepsilon\left(\bar{Z}^{i}+k\right)$ is a reference configuration of $\Omega^{i}(t), i \in\{\mathrm{a}, \mathrm{w}, \mathrm{s}\}$.

For simplicity, the geometry at $t=0$ is assumed to coincide with this reference configuration. Furthermore, the evolution of the three parts of $\Omega$ can be described by orientation-preserving mappings $\psi_{\varepsilon}^{i}(\cdot, t): \Omega_{\varepsilon}^{i} \rightarrow \Omega^{i}(t), i \in\{\mathrm{a}, \mathrm{w}, \mathrm{s}\}$, for each $t \in S=(0, T)$. Here, we have written $\Omega_{\varepsilon}^{i}$ for $\Omega^{i}(0)=\Omega_{*}^{i}$ in order to emphasise the $\varepsilon$-periodicity of the geometry. We define $\Psi_{\varepsilon}^{i}=\nabla \psi_{\varepsilon}^{i}$ and $J_{\varepsilon}^{i}=\operatorname{det} \Psi_{\varepsilon}^{i}, i \in\{\mathrm{a}, \mathrm{w}, \mathrm{s}\}$. For the sake of brevity, we write $\Gamma_{\varepsilon}=\partial \Omega_{\varepsilon}^{\mathrm{a}} \cap \partial \Omega_{\varepsilon}^{\mathrm{w}}$ and $\Gamma_{\varepsilon}^{\mathrm{ws}}=\partial \Omega_{\varepsilon}^{\mathrm{w}} \cap \partial \Omega_{\varepsilon}^{\mathrm{s}}$ and we assume $\partial \Omega_{\varepsilon}^{\mathrm{a}} \cap \partial \Omega_{\varepsilon}^{\mathrm{s}}=\emptyset$.

In order to keep things simple and to focus on the aspect of the evolution of the microstructure being induced by the reaction, only the concentrations of $\mathrm{A}$ in $\Omega_{\varepsilon}^{\mathrm{a}}$ and $\Omega_{\varepsilon}^{\mathrm{w}}$ are modelled, which we denote by $u_{\varepsilon}^{\mathrm{a}}$ and $u_{\varepsilon}^{\mathrm{w}}$, respectively. Cf. eq. (2) in [6], the reaction-diffusion system in the reference description is thus given by 


$$
\begin{array}{cl}
\partial_{t}\left(J_{\varepsilon}^{\mathrm{a}} u_{\varepsilon}^{\mathrm{a}}\right)-\nabla \cdot\left(J_{\varepsilon}^{\mathrm{a}} \Psi_{\varepsilon}^{\mathrm{a}-1} D_{\varepsilon}^{\mathrm{a}} \Psi_{\varepsilon}^{\mathrm{a}-T} \nabla u_{\varepsilon}^{\mathrm{a}}\right)=0, & x \in \Omega_{\varepsilon}^{\mathrm{a}}, t \in S, \\
\partial_{t}\left(J_{\varepsilon}^{\mathrm{w}} u_{\varepsilon}^{\mathrm{w}}\right)-\nabla \cdot\left(\varepsilon^{l} J_{\varepsilon}^{\mathrm{w}} \Psi_{\varepsilon}^{\mathrm{w}-1} D_{\varepsilon}^{\mathrm{w}} \Psi_{\varepsilon}^{\mathrm{w}-T} \nabla u_{\varepsilon}^{\mathrm{w}}\right)=-r_{\varepsilon} f_{\varepsilon}^{\mathrm{r}}, \quad x \in \Omega_{\varepsilon}^{\mathrm{w}}, t \in S, \\
-\left(J_{\varepsilon}^{\mathrm{a}} \Psi_{\varepsilon}^{\mathrm{a}-1} D_{\varepsilon}^{\mathrm{a}} \Psi_{\varepsilon}^{\mathrm{a}-T} \nabla u_{\varepsilon}^{\mathrm{a}}\right) \cdot \nu_{\varepsilon}^{\mathrm{a}}=\left(\varepsilon^{l} J_{\varepsilon}^{\mathrm{w}} \Psi_{\varepsilon}^{\mathrm{w}-1} D_{\varepsilon}^{\mathrm{w}} \Psi_{\varepsilon}^{\mathrm{w}-T} \nabla u_{\varepsilon}^{\mathrm{w}}\right) \cdot \nu_{\varepsilon}^{\mathrm{w}}, & x \in \Gamma_{\varepsilon}, t \in S, \\
-\left(J_{\varepsilon}^{\mathrm{a}} \Psi_{\varepsilon}^{\mathrm{a}-1} D_{\varepsilon}^{\mathrm{a}} \Psi_{\varepsilon}^{\mathrm{a}-T} \nabla u_{\varepsilon}^{\mathrm{a}}\right) \cdot \nu_{\varepsilon}^{\mathrm{a}}=\varepsilon a_{\varepsilon}\left\|\Psi_{\varepsilon}^{\mathrm{w}-T} \nu_{\varepsilon}^{\mathrm{w}}\right\| J_{\varepsilon}^{\mathrm{w}}\left(u_{\varepsilon}^{\mathrm{a}}-u_{\varepsilon}^{\mathrm{w}}\right), & x \in \Gamma_{\varepsilon}, t \in S, \\
-\left(\varepsilon^{l} J_{\varepsilon}^{\mathrm{w}} \Psi_{\varepsilon}^{\mathrm{w}-1} D_{\varepsilon}^{\mathrm{w}} \Psi_{\varepsilon}^{\mathrm{w}-T} \nabla u_{\varepsilon}^{\mathrm{w}}\right) \cdot \nu_{\varepsilon}^{\mathrm{w}}=0, & x \in \Gamma_{\varepsilon}^{\mathrm{ws}}, t \in S,
\end{array}
$$

together with boundary conditions at the exterior boundary as well as initial conditions. Compared to [6], we have abandoned the hats to denote functions in the reference description and we only have evolution of the domains due to deformation since we do not model concentrations in the solid matrix here.

The scaling factor $\varepsilon^{l}$ associated with $D_{\varepsilon}^{\mathrm{w}}, l \in[0,2]$, arises from a nondimensionalisation. It is related to the characteristic macroscopic and microscopic lengths as well as the diffusion times. Similar consideration motivate the scaling of the interfacial-exchange coefficient $a_{\varepsilon}$. More details on the influence and choice of different scaling exponents can be found in [10,9]. We require $\Omega_{\varepsilon}^{\mathrm{a}}$ and, if $l=0, \Omega_{\varepsilon}^{\mathrm{w}}$ to be connected.

The only functions associated with the evolution of the domains appearing in the system of equations in the reference configuration (2) are $\Psi_{\varepsilon}^{i}$ and $J_{\varepsilon}^{i}, i \in\{\mathrm{a}, \mathrm{w}\}$. We want to relate these to the reaction-diffusion process. The reaction (1) consumes a matrix constituent and produces another one. Therefore, we need to model the evolution of the subdomains depending on the reaction. For this, recall that $\Psi_{\varepsilon}^{i}$ relates the length and orientation of a material fibre in the reference configuration to its length and orientation in the current configuration and $J_{\varepsilon}^{i}$ describes the change of volume.

We consider the case where it is reasonable to assume that changes in geometry other than the volumetric changes do not have a large influence on the problem, i.e. we can assume $\Psi_{\varepsilon}^{i} \approx \mathrm{Id}, i \in\{\mathrm{a}, \mathrm{w}\}$, in the system of equations (2). For example, this is the case if nearly isotropic diffusion is always to be expected. Since it is reasonable to assume the water incompressible, we have $J_{\varepsilon}^{\mathrm{w}} \equiv 1$ for all times. Therefore, we are left with deriving an equation for $J_{\varepsilon}^{\mathrm{a}}$. Because we need to relate the change of pore-air volume to the reaction in the pore water, we only obtain a (non-local) relation of the averaged quantities.

We proceed with the derivation of an equation for $J_{\varepsilon}^{\mathrm{a}}$. The dissolution of $\mathrm{B}$ and the precipitation of $\mathrm{C}$ are assumed to be instantaneous in order to keep things simple. The reaction rate is given by a function $f_{\varepsilon}^{\mathrm{r}}(x, t)$, which is bounded independently of $\varepsilon$ in $L^{2}\left(\Omega_{\varepsilon}^{\mathrm{w}} \times S\right)$. We denote by $\tilde{f}_{\varepsilon}^{\mathrm{r}}(x, y, t)$ the unfolded version of $f_{\varepsilon}^{\mathrm{r}}$, i.e. $\tilde{f}_{\varepsilon}^{\mathrm{r}}=\mathcal{T}_{\varepsilon}\left(f_{\varepsilon}^{\mathrm{r}}\right)$ where $\mathcal{T}_{\varepsilon}$ is the periodic unfolding operator [11], and analogously $\tilde{J}_{\varepsilon}^{\mathrm{a}}$ and $\tilde{J}_{\varepsilon}^{\mathrm{w}}$. In the cell $Y$ at the point $x$, the amount (i.e. mass) of constituent $\mathrm{B}$ being used up and of constituent $\mathrm{C}$ being produced at time $t$ is given by

$$
\int_{Z^{\mathrm{w}}} m_{\mathrm{B}} \tilde{J}_{\varepsilon}^{\mathrm{w}} \tilde{f}_{\varepsilon}^{\mathrm{r}} \mathrm{d} y \text { and } \quad \int_{Z^{\mathrm{w}}} m_{\mathrm{C}} \tilde{J}_{\varepsilon}^{\mathrm{w}} \tilde{f}_{\varepsilon}^{\mathrm{r}} \mathrm{d} y
$$

respectively, where $m_{\mathrm{B}}$ and $m_{\mathrm{C}}$ are the molar weights of $\mathrm{B}$ and $\mathrm{C}$, respectively. Noting that $\tilde{J}_{\varepsilon}^{\mathrm{w}} \equiv 1$ and that an increase of total volume of constituents B and C implies a decrease of volume of pore air, we have

$$
\frac{\mathrm{d}}{\mathrm{d} t} \int_{Z^{\mathrm{a}}} \tilde{J}_{\varepsilon}^{\mathrm{a}} \mathrm{d} y=-\left|Z^{\mathrm{a}}\right| C^{\mathrm{m}} \int_{Z^{\mathrm{w}}} \tilde{f}_{\varepsilon}^{\mathrm{r}} \mathrm{d} y \quad \text { where } C^{\mathrm{m}}=\frac{1}{\left|Z^{\mathrm{a}}\right|}\left(\frac{m_{\mathrm{C}}}{\rho_{\mathrm{C}}}-\frac{m_{\mathrm{B}}}{\rho_{\mathrm{B}}}\right)
$$

for each instant in time, where $\rho_{\mathrm{B}}$ and $\rho_{\mathrm{C}}$ are the densities of constituents $\mathrm{B}$ and $\mathrm{C}$, respectively.

Since we do not expect the limit of $J_{\varepsilon}^{\mathrm{a}}$ as $\varepsilon \rightarrow 0$ to vary within one cell (and since we do not have any more information), we define $J_{\varepsilon}^{\mathrm{a}}(x, t)$ to be the constant value determined by (4) in each cell. Hence, an equation for $J_{\varepsilon}^{\mathrm{a}}$ is obtained: find $J_{\varepsilon}^{\mathrm{a}}: \Omega \times S \rightarrow \mathbb{R}$ such that

$$
\partial_{t} J_{\varepsilon}^{\mathrm{a}}(x, t)=-C^{\mathrm{m}} \int_{Z^{\mathrm{w}}} \tilde{f}_{\varepsilon}^{\mathrm{r}}(x, y, t) \mathrm{d} y, \quad J_{\varepsilon}^{\mathrm{a}}(0) \equiv 1 .
$$

The reaction-diffusion system (2) simplifies to 


$$
\begin{aligned}
& \partial_{t}\left(J_{\varepsilon}^{\mathrm{a}} u_{\varepsilon}^{\mathrm{a}}(x, t)\right)-\nabla \cdot\left(J_{\varepsilon}^{\mathrm{a}} D_{\varepsilon}^{\mathrm{a}} \nabla u_{\varepsilon}^{\mathrm{a}}\right)=0, \quad x \in \Omega_{\varepsilon}^{\mathrm{a}}, t \in S, \\
& \partial_{t} u_{\varepsilon}^{\mathrm{w}}(x, t)-\nabla \cdot\left(\varepsilon^{l} D_{\varepsilon}^{\mathrm{w}} \nabla u_{\varepsilon}^{\mathrm{w}}\right)=-r_{\varepsilon} f_{\varepsilon}^{\mathrm{r}}, \quad x \in \Omega_{\varepsilon}^{\mathrm{w}}, t \in S, \\
& -\left(J_{\varepsilon}^{\mathrm{a}} D_{\varepsilon}^{\mathrm{a}} \nabla u_{\varepsilon}^{\mathrm{a}}\right) \cdot \nu_{\varepsilon}^{\mathrm{a}}=\left(\varepsilon^{l} D_{\varepsilon}^{\mathrm{w}} \nabla u_{\varepsilon}^{\mathrm{w}}\right) \cdot \nu_{\varepsilon}^{\mathrm{w}}, \quad x \in \Gamma_{\varepsilon}, t \in S, \\
& -\left(J_{\varepsilon}^{\mathrm{a}} D_{\varepsilon}^{\mathrm{a}} \nabla u_{\varepsilon}^{\mathrm{a}}\right) \cdot \nu_{\varepsilon}^{\mathrm{a}}=\varepsilon a_{\varepsilon}\left(u_{\varepsilon}^{\mathrm{a}}-u_{\varepsilon}^{\mathrm{w}}\right), \quad x \in \Gamma_{\varepsilon}, t \in S, \\
& -\left(\varepsilon^{l} D_{\varepsilon}^{\mathrm{w}} \nabla u_{\varepsilon}^{\mathrm{w}}\right) \cdot \nu_{\varepsilon}^{\mathrm{w}}=0, \quad x \in \Gamma_{\varepsilon}^{\mathrm{ws}}, t \in S .
\end{aligned}
$$

Homogeneous Neumann boundary conditions are prescribed at the exterior boundary $\partial \Omega$ for simplicity as well as initial conditions $\left(u^{\mathrm{a}}(0), u^{\mathrm{w}}(0)\right)=\left(u_{0}^{\mathrm{a}}, u_{0}^{\mathrm{w}}\right)$.

For a function $u=u(x, t) \in L^{2}(\Omega \times S)$, let $|u(t)|_{\Omega}$ be the $L^{2}(\Omega)$-norm and $|u|_{\Omega, t}$ be the $L^{2}(\Omega \times(0, t))$ norm. For the coefficients of the system, we assume that their space variables can each be split into a macroscopic and a microscopic one (often called slow and fast component): For $\alpha \in\{\mathrm{a}, \mathrm{w}\}$, it is assumed that there exist functions $D^{\alpha}=D^{\alpha}(x, y, t), x \in \Omega_{\varepsilon}^{\alpha}, y \in Z^{\alpha}, t \in S$, bounded from above and from below by numbers $D_{\max }^{\alpha}$ and $D_{\min }^{\alpha}$, respectively, and periodically extended in $y$, such that $D_{\varepsilon}^{\alpha}(x, t)=D^{\alpha}(x, x / \varepsilon, t)$ and analogously for $a_{\varepsilon}$ and $r_{\varepsilon}$. We further assume the norm equalities

$$
\lim _{\varepsilon \rightarrow 0}\left|D_{\varepsilon}^{\alpha}(t)\right|_{\Omega}^{2}=\left|D^{\alpha}(t)\right|_{\Omega \times Z^{\alpha}}^{2}, \quad \lim _{\varepsilon \rightarrow 0}\left|r_{\varepsilon}(t)\right|_{\Omega}^{2}=|r(t)|_{\Omega \times Z^{\mathrm{w}}}^{2}, \quad \lim _{\varepsilon \rightarrow 0} \varepsilon\left|a_{\varepsilon}(t)\right|_{\Gamma_{\varepsilon}}^{2}=|a(t)|_{\Omega \times \Gamma}^{2}
$$

and $r_{\min } \geq 0$ and $D_{\min }^{\alpha}, a_{\min }>0$ as well as $\partial_{t} D_{\varepsilon}^{\alpha}, \partial_{t} a_{\varepsilon}$ bounded from above. If $C^{\mathrm{m}}>0, f_{\varepsilon}^{\mathrm{r}}$ is also required to be bounded in $L^{\infty}\left(S ; L^{2}\left(\Omega_{\varepsilon}^{\mathrm{w}}\right)\right)$.

Since the problem for $J_{\varepsilon}^{\mathrm{a}}$ is decoupled from the reaction-diffusion problem, it can be solved independently beforehand. The reaction-diffusion system is not completely standard owing to the presence of $J_{\varepsilon}^{\mathrm{a}}$ in the parabolic term. For the proof of existence of microsolutions, we make use of an abstract theory for degenerate problems developed in [12]. This enables us to prove the following theorem.

Theorem 2.1 For given $\varepsilon>0$, there exists a unique weak solution of problem (5). We have

$$
J_{\varepsilon}^{\mathrm{a}}(x, t)=1-C^{\mathrm{m}} \int_{0}^{t} \int_{Z^{\mathrm{w}}} f^{\mathrm{r}}(x, y, s) \mathrm{d} y \mathrm{~d} s,
$$

which satisfies

$$
\left|J_{\varepsilon}^{\mathrm{a}}(t)\right|_{\Omega}^{2} \leq C, \quad\left|\partial_{t} J_{\varepsilon}^{\mathrm{a}}\right|_{\Omega, t}^{2} \leq C
$$

for a.e. $t \in S$. The functions $\left(u_{\varepsilon}^{\mathrm{a}}, u_{\varepsilon}^{\mathrm{w}}\right) \in L^{2}\left(0, T ; W^{1,2}\left(\Omega_{\varepsilon}^{\mathrm{a}}\right)\right) \times L^{2}\left(0, T ; W^{1,2}\left(\Omega_{\varepsilon}^{\mathrm{w}}\right)\right)$ satisfy

$$
\left|u_{\varepsilon}^{\mathrm{a}}(t)\right|_{\Omega_{\varepsilon}^{\mathrm{a}}}+\left|\nabla u_{\varepsilon}^{\mathrm{a}}\right| \Omega_{\varepsilon}^{\mathrm{a}, t}+\left|u_{\varepsilon}^{\mathrm{w}}(t)\right|_{\Omega_{\varepsilon}^{\mathrm{w}}}+\varepsilon^{l / 2}\left|\nabla u_{\varepsilon}^{\mathrm{w}}\right| \Omega_{\varepsilon}^{\mathrm{w}}, t+\varepsilon^{1 / 2}\left|u_{\varepsilon}^{\mathrm{a}}-u_{\varepsilon}^{\mathrm{w}}\right|_{\Gamma_{\varepsilon}, t} \leq C
$$

for a.e. $t \in S$, where the constant $C$ depends on $T$ and the data but not on $\varepsilon$ if $\partial_{t} J_{\varepsilon}^{\mathrm{a}}$ is non-negative or bounded independently of $\varepsilon$ in $L^{\infty}(\Omega \times S)$.

\section{Identification of the limit problems}

For the examination of the convergence of the limit functions as $\varepsilon \rightarrow 0$ and for the identification of the limit problems, two-scale convergence $[13,14]$ and periodic unfolding [11] are used. We only present the identification of the limit problem associated with the volume factor $J_{\varepsilon}^{\mathrm{a}}$. The complete limit problem of (5) is stated in the next section.

Choosing the test function as $\varphi^{\mathrm{J}}(x, y, t)$ with $\varphi^{\mathrm{J}} \in L^{2}(\Omega \times Y \times S)$ in the weak form of (5a) and integrating over $Y$, we obtain

$$
\begin{aligned}
\int_{0}^{T} \int_{\Omega} \int_{Y} \mathcal{T}_{\varepsilon}\left(\partial_{t} J_{\varepsilon}^{\mathrm{a}}\right)(x, t) \varphi^{\mathrm{J}}(x, y, t) \mathrm{d} y & \mathrm{~d} x \mathrm{~d} t \\
& =-C^{\mathrm{m}} \int_{0}^{T} \int_{\Omega} \int_{Y} \int_{Z^{\mathrm{w}}} \mathcal{T}_{\varepsilon}\left(f_{\varepsilon}^{\mathrm{r}}\right)(x, z, t) \mathrm{d} z \varphi^{\mathrm{J}}(x, y, t) \mathrm{d} y \mathrm{~d} x \mathrm{~d} t,
\end{aligned}
$$


where we have used the fact that $J_{\varepsilon}^{\mathrm{a}}$ is constant in each cell and, therefore, $\partial_{t} J_{\varepsilon}^{\mathrm{a}}=\mathcal{T}_{\varepsilon}\left(\partial_{t} J_{\varepsilon}^{\mathrm{a}}\right)$ is independent of $y$. Since the sequences are independent of $y$, the limit equation as $\varepsilon \rightarrow 0$ is given by

$$
\int_{0}^{T} \int_{\Omega} \partial_{t} J^{\mathrm{a}}(x, t) \int_{Y} \varphi^{\mathrm{J}}(x, y, t) \mathrm{d} y \mathrm{~d} x \mathrm{~d} t=-C^{\mathrm{m}} \int_{0}^{T} \int_{\Omega} \int_{Z^{\mathrm{w}}} f^{\mathrm{r}}(x, z, t) \mathrm{d} z \int_{Y} \varphi^{\mathrm{J}}(x, y, t) \mathrm{d} y \mathrm{~d} x \mathrm{~d} t .
$$

Owing to the equivalence of convergences, these are also the two-scale limits and, moreover, it suffices to take $\varphi^{\mathrm{J}}$ independent of $y$. Therefore,

$$
\int_{0}^{T} \int_{\Omega} \partial_{t} J^{\mathrm{a}}(x, t) \varphi^{\mathrm{J}}(x, t) \mathrm{d} x \mathrm{~d} t=-C^{\mathrm{m}} \int_{0}^{T} \int_{\Omega} \int_{Z^{\mathrm{w}}} f^{\mathrm{r}}(x, y, t) \mathrm{d} y \varphi^{\mathrm{J}}(x, t) \mathrm{d} x \mathrm{~d} t
$$

for all $\varphi^{\mathrm{J}} \in L^{2}(\Omega \times S)$.

\section{The macroscopic limit problem}

We state the macroscopic limit problem of $(5)$ as $\varepsilon \rightarrow 0$, where the limit functions of $J_{\varepsilon}^{\mathrm{a}}$, $u_{\varepsilon}^{\mathrm{a}}$ and $u_{\varepsilon}^{\mathrm{w}}$ are denoted by $J^{\mathrm{a}}, u^{\mathrm{a}}$ and $u^{\mathrm{w}}$, respectively. We define a generic cell problem: Let the functions $\varsigma_{j}^{\alpha}$, $j=1, \ldots, N$, be the $Y$-periodic solution of the cell problem

$$
-\nabla_{y} \cdot\left(A(x, y, t)\left(\nabla_{y} \varsigma_{j}^{\alpha}(x, y, t)+e_{j}\right)\right)=0, \quad y \in Z^{\alpha}, x \in \Omega, t \in S,
$$

with homogeneous Neumann conditions at all interior boundaries, the weak form of which is given by

$$
\left(A(x, \cdot, t)\left(\nabla_{y} \varsigma_{j}^{\alpha}(x, \cdot, t)+e_{j}\right) \mid \nabla_{y} \phi\right)_{Z^{\alpha}}=0
$$

for all $Y$-periodic test functions $\phi$. The vector $e_{j}$ is the $j$ th unit vector in $N$-dimensional Euclidean space. Writing $\delta_{i j}$ for the Kronecker delta, the $\varsigma_{j}^{\alpha}$ are used to define the tensor $P^{\alpha}=\left[p_{i j}^{\alpha}\right]_{i j}$ as

$$
p_{i j}^{\alpha}(x, t)=\int_{Z^{\mathrm{a}}} A(x, y, t)\left(\delta_{i j}+\partial_{y_{i}} \varsigma_{j}^{\alpha}(x, y, t)\right) \mathrm{d} y .
$$

We will specify $A$ and $\alpha$ to our needs below.

The limit problem for $J^{\text {a }}$ is given by

$$
\partial_{t} J^{\mathrm{a}}(x, t)=-C^{\mathrm{m}} \int_{Z^{\mathrm{w}}} f^{\mathrm{r}} \mathrm{d} y, \quad x \in \Omega, t \in S,
$$

with initial condition $J^{\mathrm{a}}(0)=1$. The limit equation for $u^{\mathrm{a}}$ states

$$
\partial_{t}\left(J^{\mathrm{a}} u^{\mathrm{a}}(x, t)\right)-\nabla \cdot\left(P^{\mathrm{a}} \nabla u^{\mathrm{a}}\right)=-\int_{\Gamma} a\left(u^{\mathrm{a}}-u^{\mathrm{w}}\right) \mathrm{d} \sigma_{y}, \quad x \in \Omega, t \in S,
$$

with homogeneneous Neumann conditions at $\partial \Omega$ and initial condition $u^{\mathrm{a}}(0)=u_{0}^{\mathrm{a}}$, where $P^{\mathrm{a}}$ is the tensor defined by (15) with $A=J^{\mathrm{a}} D^{\mathrm{a}}$ and $\alpha=$ a.

The limit equation for $u^{\mathrm{w}}$ depends on the particular choice of the scaling exponent $l$. If $l<2$, the limit equation is given by

$$
\partial_{t} u^{\mathrm{w}}(x, t)-\delta_{0 l} \nabla \cdot\left(P^{\mathrm{w}} \nabla u^{\mathrm{w}}\right)=-\int_{Z^{\mathrm{w}}} r f^{\mathrm{r}} \mathrm{d} y+\int_{\Gamma} a\left(u^{\mathrm{a}}-u^{\mathrm{w}}\right) \mathrm{d} \sigma_{y}, x \in \Omega, t \in S,
$$

where $P^{\mathrm{w}}$ is defined by (15) with $A=D^{\mathrm{w}}$ and $\alpha=\mathrm{w}$. If $l=2$, the limit problem is given by

$$
\begin{aligned}
\partial_{t} u^{\mathrm{w}}(x, y, t)- & \nabla_{y} \cdot\left(D^{\mathrm{w}} \nabla_{y} u^{\mathrm{w}}\right)=-r f^{\mathrm{r}}, & & x \in \Omega, y \in Z^{\mathrm{w}}, t \in S, \\
- & D^{\mathrm{w}} \nabla_{y} u^{\mathrm{w}} \cdot \nu^{\mathrm{w}}=a\left(u^{\mathrm{a}}-u^{\mathrm{w}}\right), & & x \in \Omega, y \in \Gamma, t \in S, \\
- & D^{\mathrm{w}} \nabla_{y} u^{\mathrm{w}} \cdot \nu^{\mathrm{w}}=0, & & x \in \Omega, y \in \Gamma^{\mathrm{ws}}, t \in S .
\end{aligned}
$$

For all choices of $l$, homogeneous Neumann conditions apply at the exterior boundaries and $u^{\mathrm{w}}(0)=u_{0}^{\mathrm{w}}$. 
Thus, the limit problem consists of one ordinary differential equation for the macroscopic pore-air volume factor $J^{\mathrm{a}}(x, t)$ coupled to a reaction-diffusion problem for the concentrations $u^{\mathrm{a}}$ and and $u^{\mathrm{w}}$, where we have $u^{\mathrm{a}}=u^{\mathrm{a}}(x, t)$ as well as $u^{\mathrm{w}}=u^{\mathrm{w}}(x, t)$ if $l<2$ and $u^{\mathrm{w}}=u^{\mathrm{w}}(x, y, t)$ if $l=2$.

\section{Acknowledgements}

The great support of M. Böhm and the fruitful discussions with S. A. Meier from the University of Bremen and W. Jäger from the University of Heidelberg during this research are thankfully appreciated. Furthermore, the author is grateful for the financial support of the German National Academic Foundation.

\section{References}

[1] J. Kropp, Relations between transport characteristics and durability, Performance criteria for concrete durability, RILEM Report 12 (1995), 97-137.

[2] T. A. Bier, Karbonatisierung und Realkalisierung von Zementstein und Beton, PhD dissertation, University of Karlsruhe (1988).

[3] A. Muntean, A moving-boundary problem: modeling, analysis and simulation of concrete carbonation, PhD dissertation, University of Bremen, Germany, 2006. Also: Cuvillier Verlag, 2006.

[4] S. A. Meier, M. A. Peter, M. Böhm, A two-scale modelling approach to reaction-diffusion processes in porous materials, Comp. Mat. Sci. 39 (2007), 29-34.

[5] S. A. Meier, M. A. Peter, A. Muntean, M. Böhm, J. Kropp, A two-scale approach to concrete carbonation, Proc. International RILEM Workshop on Integral Service Life Modelling of Concrete Structures, 5-6 November 2007, Guimarães, Portugal (in press).

[6] M. A. Peter, Homogenisation in domains with evolving microstructure, C. R. Mécanique 335(7) (2007), 357-362.

[7] A. Bensoussan, J. L. Lions, G. Papanicolaou, Asymptotic analysis for periodic structures, North-Holland, 1978.

[8] E. Sanchez-Palencia, Non-homogeneous media and vibration theory, Springer, 1980.

[9] M. A. Peter, Coupled reaction-diffusion systems and evolving microstructure: mathematical modelling and homogenisation, PhD dissertation, University of Bremen, Germany, 2006. Also: Logos Verlag Berlin, 2007.

[10] M. A. Peter, M. Böhm, Scalings in homogenisation of reaction, diffusion and interfacial exchange in a two-phase medium, Proc. Equadiff-11 (ed. by M. Fila, A. Handlovicova, K. Mikula, M. Medved, P. Quittner and D. Sevcovic), Bratislava, SK (2005), 369-376.

[11] D. Cioranescu, A. Damlamian, G. Griso, Periodic unfolding and homogenization, C. R. Acad. Sci. Paris, Ser. I 335 (2002), 99-104.

[12] R. E. Showalter, Monotone operators in Banach space and nonlinear partial differential equations, American Mathematical Society, 1997.

[13] G. Nguetseng, A general convergence result for a functional related to the theory of homogenization, SIAM J. Math. Anal. 20(3) (1989), 608-629.

[14] G. Allaire, Homogenization and two-scale convergence, SIAM J. Math. Anal. 23(6) (1992), 1482-1518. 